\title{
Atomically Resolved Local Structure of Conductive Domain Walls in Ferroelectric $\mathrm{BiFeO}_{3}$.
}

\author{
A. Bencan ${ }^{1}$, G. Drazic ${ }^{2}$, H. Ursic ${ }^{1}$, N. Sakamoto ${ }^{3}$, B. Jancar ${ }^{4}$, G.Tavcar $^{5}$, M. Makarovic ${ }^{1}$, J. Walker $^{1}$, B. \\ Malic $^{1}$,D. Damjanovic ${ }^{6}$ and T. Rojac ${ }^{1}$ \\ 1. Electronic Ceramics Department, Jozef Stefan Institute, Ljubljana, Slovenia. \\ 2. Laboratory for Materials Chemistry, National Institute of Chemistry, Ljubljana, Slovenia. \\ 3. Research Institute of Electronics, Shizuoka University, Hamamatsu, Japan. \\ 4. Advanced Materials Department, Jozef Stefan Institute, Ljubljana, Slovenia. \\ 5. Department of Inorganic Chemistry and Technology, Jozef Stefan Institute, 1000 Ljubljana, Slovenia \\ 6. Ceramics Laboratory, Swiss Federal Institute of Technology - EPFL, Switzerland.
}

The functional responses of ferroelectrics are inherently influenced by their crystal and domain structure. We recently demonstrated that the multiferroic $\mathrm{BiFeO}_{3}(\mathrm{BFO})$ ceramics possess domain walls (DWs) which are more conductive then domains themselves [1]. In order to make further steps toward understanding of the DW conductivity, we precisely analysed the local structure, such as the local variations in lattice parameters, strain gradients and off-center Fe displacements across different DWs at the atomic level.

$\mathrm{BiFeO}_{3}$ ceramics were prepared by reactive sintering of a mechanochemically activated $\mathrm{Bi}_{2} \mathrm{O}_{3}-\mathrm{Fe}_{2} \mathrm{O}_{3}$ powder mixture at $760^{\circ} \mathrm{C}$ or $820^{\circ} \mathrm{C}$ in a packing powder. The samples for the STEM analyses were prepared using standard methods; after cutting, the samples were ground, polished down to $100 \mu \mathrm{m}$ and, after dimpling, finally thinned down to electron transparency using Gatan PIPS ion-milling system. The structure of domain walls in the BFO ceramics was analysed by atomically resolved Cs-probe corrected JEM-ARM 200CF STEM microscope operated at $200 \mathrm{kV}$.

Rhombohedral BFO has three different DWs, labelled according to the angle between the $[111]_{\mathrm{pc}}$ polarization vectors in adjacent domains, i.e., $180^{\circ} \mathrm{DW}$ parallel to $\{111\}_{\mathrm{pc}}$ planes, $71^{\circ} \mathrm{DW}$ parallel to $\{110\}_{\text {pc }}$ planes and $109^{\circ}$ DW parallel to $\{100\}_{\text {pc }}$ planes. DWs in BFO are not flat and show steps (see Fig 1). A detailed analysis of a $109^{\circ} \mathrm{DW}$ is shown in Fig. 2. From HAADF STEM images we measured the displacement of $\mathrm{Fe}$ cations from the center of the periodic Bi sub-lattice and were able to determine the position and the type of the DW.

$109^{\circ}$ domain wall is parallel to $\{100\}_{\text {pc }}$ planes and via steps change from head-to-head (charged) to head-to tail (non-charged). This is seen in Fig 2a by the change of the plane of the DW from (100) pc to $(010)_{\text {pc }}$. The width of the non-charged wall is $\sim 7$ unit cells; the width of the charged domain wall is $\sim 15$ unit cells. Although charged domain walls are thicker compared to the non-charged, the difference is not an order of magnitude as calculated by Gureev et al. [2]. The results indicate that although the DWs appear head-to-tail on a micron scale (e.g., by SEM imaging), they show, in fact, local steps on the atomic level with head-to-head (charged) configurations.

The calculated Fe displacement map made from $109^{\circ}$ DW (Fig. 2a) is shown in Fig 2b. Arrows show the magnitude and the direction of Fe displacement, i.e., the longer is the arrow, the larger is the displacement. The displacements of the Fe atom from the central position on $109^{\circ} \mathrm{DWs}$ are small. In the 
presentation, possible origins contributing to the above-described variations at DWs will be discussed [3].

\section{References:}

[1] T. Rojac et al, Adv. Funct. Mater. 25 (2015), p. 2099.

[2] M.Y. Gureev, A.K. Tagantsev and N. Setter, Phy. Rev. B 83 (2011), p. 184104.

[3] This work was supported by the Slovenian Research Agency (P2-0105 and J2-5483).
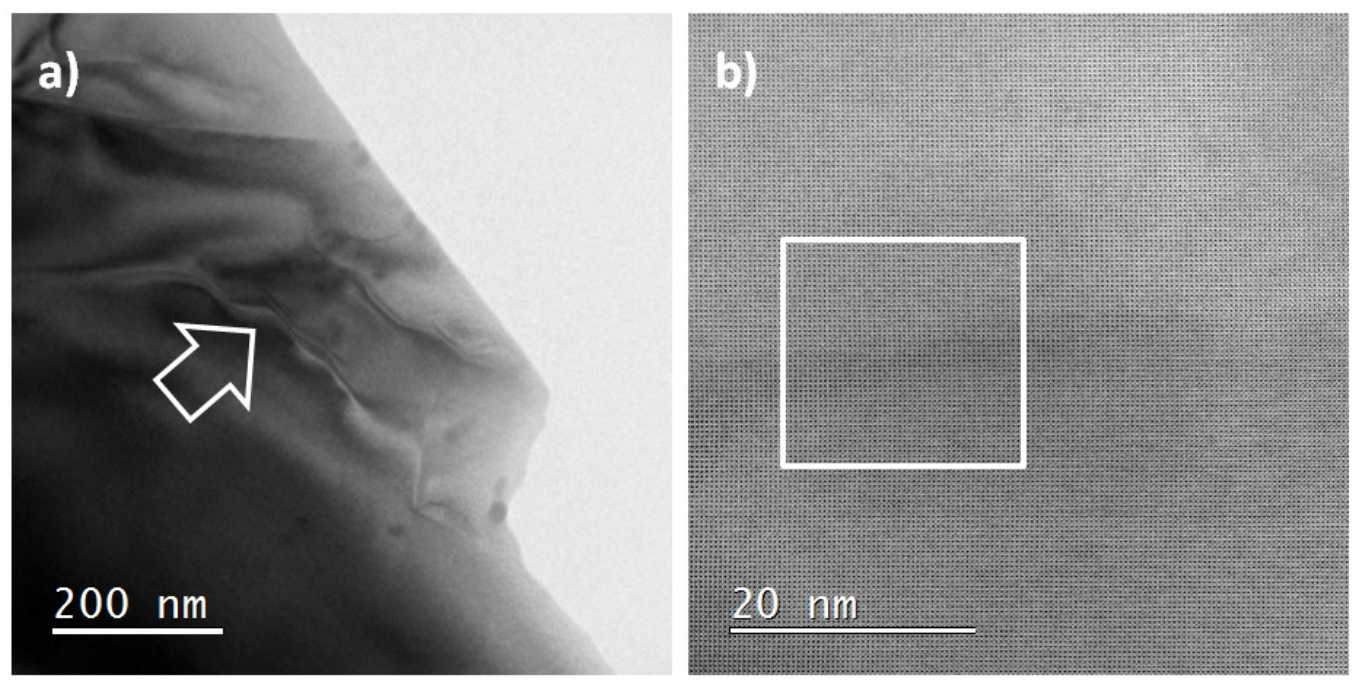

Figure 1. TEM (a) and BF-STEM (b) image of step-like DW in $\mathrm{BiFeO}_{3}$ ceramics.
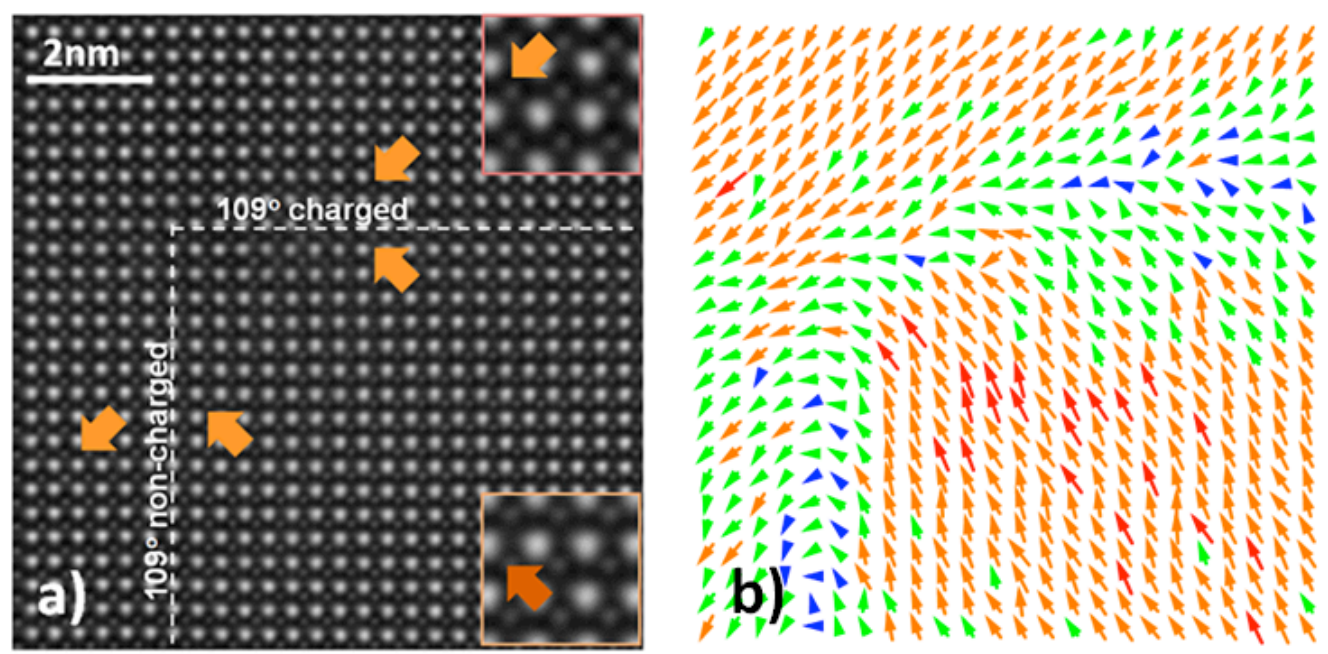

Figure 2. HAADF-STEM image of step-like $109^{\circ} \mathrm{DW}$ (a). The wall is parallel to $\{100\}_{\mathrm{pc}}$ planes and it changes via a step from charged (head/head) to non-charged (head/tail). The DW is marked with a thin dotted line. Inserts show Fe displacement from the center of the Bi sub-lattice in respective domains. Fedisplacement map of the region shown in Fig. a. (b) Arrows length and colors show the magnitude and the direction of the Fe displacement from the center of the Bi sub-lattice. 